\title{
¿Es la doble jornada igual para todas? Distribución y factores determinantes del trabajo doméstico y extradoméstico entre madres jefas de hogar y madres esposas
}

\author{
Andrea Bautista León \\ Gabriela Sánchez Soto \\ Universidad de Texas en San Antonio
}

Recepción: 13 agosto de 2016 Aceptación: 25 abril de 2017

Resumen Esta investigación provee un análisis de la desigual carga de trabajo doméstico y sus determinantes entre madres jefas de hogar y madres esposas de jefes de hogar. Analizamos: ¿Cuál es la diferencia en las horas de trabajo doméstico entre madres esposas y jefas de hogar? ¿Cuáles son los determinantes sociodemográficos de estas diferencias? ¿Influye el tipo de ocupación en la carga de trabajo doméstico? Utilizando información de uso de tiempo, comparamos las horas dedicadas al trabajo doméstico por las esposas con las que le dedican las jefas de hogar. Los resultados muestran que la carga de trabajo doméstico es cuatro horas mayor para las madres esposas y que entre los determinantes más importantes están la edad de las madres, el tipo de ocupación y las condiciones laborales.

PALABRAS CLAVE: doble jornada, trabajo doméstico, trabajo extradoméstico, conflicto trabajofamilia, desigualdad de género.

Abstract This research provides an analysis of the unequal distribution of domestic work and its determinants among mothers who are heads of household and mothers who are wives of the householder. This paper analyzes, what is the difference in the hours of domestic work between wives and female householders? What are the socio-demographic determinants of these differences? And, whether type of occupation has an impact on domestic workload? We use time-use data to compare the number of hours of domestic work of wives and householders. First, we find that domestic workload is four hours greater for wives than for householders; second, some of the most important determinants of domestic workload are age, type of occupation, and working conditions.

KEYWORDS: second-shift, domestic work, extra-domestic work, work-family conflict, gender inequality. 


\section{Introducción}

I nvestigaciones recientes acerca de la carga total de trabajo doméstico y extradoméstico han demostrado que, a diferencia de lo que ocurre entre los homno disminuye su carga de trabajo doméstico. En la presente investigación señalamos que esta sobrecarga de trabajo es desigual no solo cuando se les compara con los hombres, sino también si se les compara entre ellas mismas. Esta desigualdad proviene de las relaciones de género que se viven diariamente en los hogares y se manifiesta en diferentes grados de acuerdo con la edad, el tipo de trabajo remunerado y el estado civil de las mujeres. Considerando lo anterior, esta investigación provee un análisis de la carga de trabajo doméstico y sus determinantes entre mujeres mexicanas madres que se declararon jefas de hogar y madres cónyuges o parejas del jefe. El estudio de estos temas es relevante pues añade elementos a varias discusiones que se presentan hoy en día acerca del conflicto familia-trabajo; a las formas en que las mujeres acceden al poder y a la toma de decisiones en sus hogares, y al bienestar de las mujeres en términos de salud física, psicológica y su interacción social.

El presente trabajo se divide en una introducción teórica acerca del cambio en la participación de las mujeres mexicanas en el trabajo remunerado o extradoméstico ${ }^{1}$ y una discusión acerca de los hogares con jefatura femenina y su distinción respecto de los hogares nucleares. Más adelante se presenta una sección metodológica donde se explican la estrategia y la construcción de variables utilizadas, seguida por los resultados del análisis empírico. Posteriormente se muestran las conclusiones del trabajo.

\section{La doble jornada en las mujeres mexicanas}

La reproducción cotidiana de la vida familiar y de la sociedad comprende tanto la participación de sus miembros en la producción de mercancías, bienes y servicios como la realización de trabajo doméstico. En ambas esferas de reproducción social existe una división sexual de las labores que se consideran responsabilidad propia de un género y no del otro (Buchmann, Kriesi, Pfeifer \& Sacchi, 2003). Esta diferenciación de actividades y labores de acuerdo con el sexo de los individuos es un fenómeno que ha llamado la atención en décadas pasadas por sus efectos en el bienestar de las mujeres dado el aumento de su participación en el trabajo remunerado.

1 En esta investigación el término trabajo remunerado o extradoméstico se usa de manera indistinta para referir aquellas actividades de intercambio de fuerza de trabajo por una compensación económica. 
Hochschild y Machung (2012) introdujeron el término "doble jornada", o second shift en inglés, a finales de la década de los ochenta del siglo pasado, que refiere a la carga de trabajo que tienen las mujeres en sus hogares además de la que tienen en sus trabajos remunerados. Las autoras narran lo que ocurre en el contexto estadounidense, pero está comprobado que es un fenómeno presente a escala mundial en diversos contextos. Recientemente se han hecho esfuerzos para construir definiciones que midan y capturen esta situación.

En el caso de México, en décadas recientes se ha presentado un incremento en el volumen y la proporción de mujeres que realizan algún tipo de trabajo remunerado. De acuerdo con estimaciones propias hechas con base en la serie histórica censal del Instituto Nacional de Estadística y Geografía (INEGI, 2010), la proporción de mujeres que contaban con un trabajo remunerado o se encontraban en busca de uno (clasificadas como población económicamente activa) en 1990 fue de $19.6 \%$, en 2000 de $29.9 \%$, en 2010 de $33.3 \%$ y en 2015 alcanzó un $37.7 \%$. Este crecimiento en la participación de las mujeres en el trabajo remunerado está asociado a varias circunstancias, entre ellas el aumento de la escolaridad de esta población (Zabludovsky, 2007), y en otras sirve como estrategia familiar para incrementar el ingreso económico, y por ende el poder adquisitivo (García \& Pacheco, 2000). Si bien algunas mujeres se insertan en el mercado de trabajo remunerado en un principio por razones vinculadas con algún grado de vulnerabilidad, como necesidad económica o crisis familiares, una vez que se insertan en la fuerza laboral sus expectativas cambian y hacen proyectos a futuro independientemente de la crianza de los hijos. De igual manera, el trabajo remunerado les da elementos de identidad personal que van cambiando también su percepción de las relaciones de poder en la familia y la distribución del trabajo doméstico (Castellanos Lázaro, Zapata Martelo \& Martínez Corona, 2007).

Es importante mencionar que si bien existe una división sexual del trabajo remunerado y algunas actividades se consideran socialmente más aceptadas como femeninas que otras, en la actualidad, en México las mujeres participan en diferentes niveles de ocupaciones en el mercado de trabajo remunerado. Sin embargo, la investigación académica reciente ha demostrado que este incremento en la participación en el trabajo remunerado no ha estado vinculado con la distribución equitativa del trabajo doméstico entre los miembros del hogar y que recae sobre todo en las mujeres (Santoyo \& Pacheco, 2014). Aunado a este aumento en la participación y heterogeneidad de ocupaciones de las mujeres en el trabajo remunerado, se ha suscitado un aumento en la proporción de los hogares liderados por mujeres en las últimas décadas. Por ello consideramos necesario abordar el tema de la desigualdad en la distribución de las tareas domésticas no solo en los hogares nucleares sino entre otros tipos de hogares, además de explorar las ocupaciones remuneradas en las que se encuentran estas mujeres trabajando y observar si algunas son más favo- 
rables que otras en la disminución de la carga de trabajo doméstico.

La siguiente sección provee una exploración de la heterogeneidad de hogares mexicanos que existe en la actualidad.

\section{Cambios en la familia mexicana}

A partir de la segunda mitad del siglo xx se intensifica el crecimiento de arreglos de vida familiar no tradicionales diferentes de los hogares nucleares (Cuevas Hernández, 2010); se da principalmente el surgimiento de los hogares monoparentales, donde solo está presente el padre o la madre. Además de estos tipos de hogares, es posible identificar hogares extensos donde habitan, aparte de padres e hijos, otros miembros como pueden ser abuelos o tíos, las parejas homosexuales y las familias reconstituidas o polinucleares, en las que uno o los dos cónyuges provienen de alguna unión marital anterior y suelen tener la tutela de los hijos (Valdivia Sánchez, 2008).

En esta investigación nos centraremos en el caso de los hogares monoparentales dirigidos por mujeres que son madres y no viven con una pareja para comparar su carga de trabajo doméstico con la de esposas y madres que viven en hogares nucleares. A este respecto, es importante mencionar que para el año 2015 se estimó que alrededor del $70 \%$ de las madres que viven sin un cónyuge se declararon solteras, separadas o divorciadas, mientras que el 30 \% restante como viudas. Esta situación tiene sentido en un contexto actual donde ha ocurrido un aumento en la proporción de separaciones o divorcios. De acuerdo con el INEGI, en 1980 se registraron cuatro divorcios por cada cien matrimonios, mientras que en 2013 ascendió a 19 el número de separaciones por cada cien matrimonios en todo México (INEGI, 2016).

Respecto a las madres que son esposas y viven con una pareja, la investigación ha mostrado que estas sienten una doble responsabilidad de cuidado, de los hijos y del esposo; también perciben que reciben poco apoyo de sus parejas en las labores domésticas, lo cual no solo refleja falta de ayuda en la vida cotidiana, sino un aspecto de desigualdad proveniente de la estructura familiar tradicional que en última instancia limita el proyecto de vida de las mujeres (Álvarez \& Gómez, 2011).

En relación con las madres jefas de hogar, la investigación se divide principalmente en tres enfoques. Primero, desde una perspectiva de exclusión y precariedad, en el que estas mujeres se caracterizan por su menor cantidad de ingresos económicos por tener que participar activamente en el trabajo extradoméstico y porque muchas veces no cuentan con ayuda de familiares para la crianza de sus hijos (Jociles, Monco, Villamil \& Díaz, 2008; Ochoa Ávalos, 2007). Segundo, estudia esta población desde un enfoque de crítica a la estigmatización social que se tiene hacia las madres solteras y los roles sociales que deben cumplir (Oliveira \& García, 2004; Guarriello Heath, 2016). Un tercero, que comprende estudios acerca de las 
madres que se declaran jefas de hogar, ha mostrado que para algunas mujeres la ausencia del cónyuge deriva en menor carga de trabajo doméstico y aumento del tiempo dedicado a amigos o familia (Castellanos Lázaro, Zapata Martelo \& Martínez Corona, 2007). Además, otros autores señalan que las familias dirigidas por mujeres no necesariamente son las más pobres y representan casi un cuarto del total de los hogares de México en 2005 (Cuevas Hernández, 2010; Farías Anda, Salazar Segura, Monjardín Nieto \& Calleja Bello, 2014). Las mujeres jefas de hogar recurren a redes de apoyo principalmente de familiares y a otras mujeres (madres, hermanas y abuelas), en quienes delegan el cuidado de menores, o a la contratación de personal doméstico. Por lo anterior, en los hogares con jefatura femenina existe mayor equidad en la distribución del trabajo doméstico y en la toma de decisiones respecto a la repartición del dinero, así como menores índices de violencia familiar (Farías Anda, Salazar Segura, Monjardin Nieto \& Calleja Bello, 2014).

Entonces, con los factores mencionados, es posible deducir que la carga de trabajo doméstico se da en forma diferenciada entre las poblaciones que se estudian. Para apoyar este argumento, la investigación de Nieto (2004) da cuenta de que la mayor carga de trabajo doméstico recae en las mujeres que se declaran cónyuges del jefe de hogar y es en promedio de 60 horas a la semana, mientras que para las jefas de hogar el promedio fue de casi 44 horas. Sin embargo, otro de sus hallazgos resalta que la participación de las jefas de hogar en el trabajo extradoméstico es superior a la participación del promedio de las mujeres. En esta investigación se presenta el cálculo de la carga global, que incluye el trabajo doméstico y el extradoméstico en un primer análisis, para después explorar los factores sociodemográficos y de la ocupación remunerada que influyen en esta carga de trabajo.

\section{Estrategia metodológica}

En la presente investigación se estudia el año 2015, en el cual 10,174,808 millones de mujeres reportaron ser madres y estar realizando algún trabajo remunerado. Esta cantidad es igual al $54.1 \%$ de la población de mujeres que realiza trabajo remunerado. De dicha población, el 29.3 \% corresponde a madres que se declararon jefas de hogar y no viven con una pareja, mientras que el $70.7 \%$ restante son madres trabajadoras que se identificaron como cónyuges del jefe de hogar (INEGI, 2015). Considerando que la investigación previa ha mostrado que la carga de trabajo doméstico es desigual para las madres jefas de hogar y las esposas, seleccionar estas poblaciones nos permitirá contestar las siguientes preguntas: ¿qué tan amplia es esta diferencia en las horas de trabajo doméstico entre las madres esposas y las jefas de hogar?, ¿cuáles son los determinantes sociodemográficos de las diferencias en la repartición del trabajo doméstico entre estas poblaciones?, ¿el tipo de ocupación en 
el trabajo remunerado que las madres realizan influye en la carga de trabajo doméstico? Como hipótesis de trabajo, se considera que las madres esposas tendrán una mayor carga de trabajo doméstico que las madres jefas de hogar sin cónyuge. En el mercado de trabajo remunerado existen diferentes ocupaciones favorables para el balance de la vida familiar.

El análisis de estas dos poblaciones se realizó con información del primer trimestre de 2015 de la Encuesta Nacional de Ocupación y Empleo (ENOE) levantada por el INEGI y tiene como objetivo dar cuenta de la situación ocupacional del país. Cuenta con un diseño de muestreo probabilístico, bietápico estratificado y por conglomerados, la unidad para la conformación de la muestra es la vivienda (INEGI, 2007). La ENOE se ha levantado continuamente desde 2005 y es representativa a nivel nacional. Se seleccionó el primer trimestre pues contiene información sobre el uso del tiempo de los individuos encuestados.

Las mujeres seleccionadas para esta investigación son aquellas que corresponden a alguno de los dos grupos de interés: madres jefas de hogar viviendo sin pareja/cónyuge o madres esposas. El primer grupo se compone de las que se declararon solteras, divorciadas, separadas o viudas $(31.4 \%, 13.4 \%, 28.3 \%$ y $26.8 \%$ respectivamente), que contaban con al menos un hijo, se encontraban trabajando en la semana anterior a la encuesta y se declararon jefas de familia. En este grupo es importante hacer notar que en su composición incluye mujeres que tuvieron la transición de un hogar nuclear - por separación, divorcio o viudez- a uno monoparental o a uno extenso; la investigación respecto a estas últimas indica que las mujeres que se declaran jefas de hogar aun viviendo en hogares extensos es porque ellas les proveen la mayor parte de los recursos económicos y por ende cuentan con un importante poder de decisión (Farías Anda, Salazar Segura, Monjardín Nieto \& Calleja Bello, 2014). El segundo grupo se compone de mujeres que se declararon cónyuges o vivían en unión libre con el jefe de hogar, tienen al menos un hijo y se encontraban trabajando en la semana del levantamiento.

En el análisis exploratorio, para tener una idea más clara de la magnitud del trabajo doméstico y extradoméstico realizado por las mujeres de la muestra, y especialmente para crear un marco de referencia por medio del cual comparar la participación en trabajo doméstico y extradoméstico de las madres esposas, utilizamos información sobre hombres unidos en la muestra de la ENOE. A este respecto se encontraron dos limitantes: no fue posible identificar a los cónyuges o parejas de las madres esposas, y tampoco se puede saber si los hombres de la encuesta tienen hijos. Estos problemas se solucionaron seleccionando a un grupo de hombres unidos o casados que se declararon jefes de hogar y habitaban hogares donde vivían menores de 12 años, los cuales se asumen muy probablemente como sus hijos y que por el ciclo de vida de ellos requerirían un mayor tiempo de cuidados. Lo cual los hace un grupo ideal para comparar la carga de trabajo global de ellos con los dos grupos de mujeres. 
Con información del módulo sobre el uso del tiempo, se identificaron las horas dedicadas a diversas actividades que involucran el trabajo doméstico, como lo son: cuidar sin remuneración económica a niños, ancianos, enfermos o discapacitados; realizar compras, llevar las cuentas o realizar trámites para el hogar o encargarse de la seguridad; llevar a algún miembro del hogar a la escuela, cita médica u otra actividad; realizar los quehaceres de su hogar; construcción y mantenimiento de la vivienda. Con esta información se codificó una variable que captura el total de horas dedicadas a cada una de estas actividades. Por otra parte, se calculó el número total de horas en trabajo extradoméstico que realizaron estas mujeres y el grupo de hombres en la semana de referencia.

El primer análisis, para el cual también se muestran los resultados de los varones, compara el promedio de horas dedicadas a diferentes actividades entre las dos poblaciones seleccionadas. El análisis se hizo con una prueba $t$, asumiendo varianzas iguales; esta prueba sirve para identificar si las diferencias entre las medias de las dos poblaciones son significativas (Nicholson, 2014). Las variables incluidas para comparar a las poblaciones fueron: horas dedicadas a cuidados, compras, mandados, quehaceres construcción y mantenimiento de la vivienda; horas dedicadas a trabajo extradoméstico; el ingreso por hora en el trabajo extradoméstico; el porcentaje de población con seguridad social por su empleo; la edad, el número de hijos y el número de personas que viven en el hogar.

Posteriormente se estimó un modelo de regresión lineal para explicar la cantidad de horas dedicadas al trabajo doméstico, con base en las características de cada población de mujeres. Debido a que el tamaño de la muestra es diferente para cada una de ellas (madres solteras $n=10,538$ y madres esposas $n=25,227$ ) se decidió estandarizar el número de horas dedicadas a trabajo doméstico, esto es, transformar la variable para que tenga los valores de media cero y desviación estándar igual a uno. De esta manera, los coeficientes de las regresiones son comparables e interpretables entre ambas muestras.

\section{Resultados}

\section{Desigualdad en la carga de trabajo}

Para comprender cómo es que las mujeres viven la doble jornada día con día es necesario estudiar la distribución del tiempo que dedican al trabajo doméstico y al extradoméstico. Comparaciones internacionales muestran que, en promedio, las mujeres pasan 20 horas en la realización del trabajo doméstico, comparadas con un promedio de entre siete y nueve horas de los hombres (Baxter \& Tai, 2016), lo 
cual tiene implicaciones en el tiempo libre que pueden tener, el conflicto entre el trabajo y la familia y la felicidad en general, lo que afecta el bienestar (Connerley \& Wu, 2016). El cuadro 1 muestra los resultados obtenidos para México, los cuales son similares a los datos obtenidos en el documento antes citado.

Si bien los varones tienen más horas de trabajo remunerado, se observa que su carga global es de 57.1 horas, y es más baja que la de las madres jefas de hogar (65.1) y la de las madres esposas (69.4). El número de horas de trabajo doméstico de los jefes de hogar es similar a los números encontrados en otras investigaciones, como la mencionada anteriormente, pues asciende a 8.8 horas, mientras que en el caso de las mujeres las horas para ambas poblaciones de madres mexicanas exceden las proporciones vistas en el trabajo de Baxter y Tai (2016), que son 26.8 para las jefas de hogar y 33.7 para las madres esposas.

En el caso de la distribución del tiempo entre los varones y las mujeres, resaltan las actividades de cuidados y de quehaceres para los tres grupos. Por otra parte, es notable el hecho de que las madres esposas dedican el doble de tiempo a las labores de cuidado en comparación con los varones, y que en el caso de los quehaceres ellos dedican solo un poco menos de un tercio de lo que las madres jefas y madres esposas dedican cotidianamente.

El nivel de escolaridad de las mujeres (9.0 y 10.0 respectivamente) y de los hombres (9.7) es similar y corresponde al nivel de secundaria concluida. Este elemento es importante; la investigación de Casique (2008) muestra que por cada año adicional de educación hay un incremento en el trabajo doméstico reportado por las mujeres, aunque cuando se analizan parejas cada año adicional de escolaridad se incrementa la participación de los hombres en el trabajo doméstico.

Cuando se comparó el ingreso por hora que reciben las poblaciones de mujeres no se encontraron diferencias significativas, lo cual puede implicar que en promedio no hay diferencias pronunciadas en el tipo de trabajo remunerado que desarrollan. La edad es un factor importante considerando el ciclo de vida en el cual se encuentran estas poblaciones. Los resultados de la comparación de medias indican que las madres solteras de la muestra tienen en promedio seis años más de edad. El número de hijos de las madres solteras es ligeramente mayor en promedio (3.1) que el de las madres esposas (2.8). Estudiar el tamaño del hogar donde viven estas poblaciones nos ayuda a aproximarnos al número de personas que podrían ayudar en la realización del trabajo doméstico, así como a la cantidad de trabajo que se requiere en el hogar. Aunque el tamaño del hogar no sea necesariamente proporcional a la carga doméstica, se considera que entre más grande sea este es más probable que haya miembros con mayor dependencia de cuidados, como niños o personas adultas mayores. En este caso los hogares de las madres solteras son en promedio más pequeños (3.2) que el de las madres esposas (4.3). 
CUADRO 1. Diferencia de medias en la carga global de trabajo y otras características entre madres jefas de hogar y madres esposas e información de los jefes de hogar

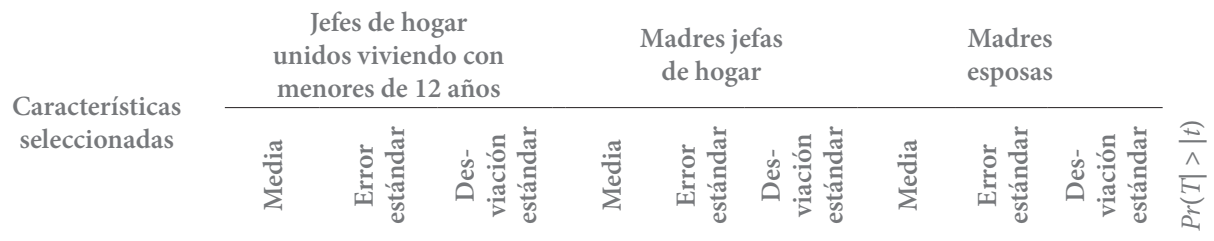

\begin{tabular}{|c|c|c|c|c|c|c|c|c|c|c|}
\hline $\begin{array}{l}\text { Horas carga de } \\
\text { trabajo total }\end{array}$ & 57.12 & 56.935 & 57.306 & 65.13 & 0.197 & 19.906 & 69.41 & 0.127 & 19.916 & * \\
\hline $\begin{array}{l}\text { Horas totales de } \\
\text { trabajo doméstico }\end{array}$ & 8.82 & 10.283 & 8.717 & 26.78 & 0.140 & 14.414 & 33.67 & 0.104 & 16.607 & * \\
\hline $\begin{array}{l}\text { Dedicadas a } \\
\text { cuidados de niños, } \\
\text { ancianos, enfermos } \\
\text { o discapacitados }\end{array}$ & 3.32 & 0.034 & 6.470 & 4.48 & 0.088 & 9.060 & 7.81 & 0.073 & 11.679 & $*$ \\
\hline $\begin{array}{l}\text { Dedicadas a } \\
\text { compras y trámites }\end{array}$ & 1.41 & 0.011 & 2.141 & 2.66 & 0.026 & 2.670 & 2.87 & 0.017 & 2.668 & * \\
\hline $\begin{array}{l}\text { Dedicadas a } \\
\text { mandados }\end{array}$ & 0.45 & 0.008 & 1.520 & 0.63 & 0.018 & 1.815 & 0.94 & 0.014 & 2.189 & * \\
\hline $\begin{array}{l}\text { Dedicadas a } \\
\text { quehaceres }\end{array}$ & 3.05 & 0.023 & 4.437 & 18.94 & 0.093 & 9.616 & 22.00 & 0.064 & 10.180 & $*$ \\
\hline $\begin{array}{l}\text { Dedicadas a } \\
\text { construir o ampliar } \\
\text { su vivienda }\end{array}$ & 0.05 & 0.004 & 0.782 & 0.00 & 0.000 & 0.043 & 0.00 & 0.002 & 0.248 & \\
\hline $\begin{array}{l}\text { Dedicadas a reparar } \\
\text { o mantenimiento a } \\
\text { la vivienda y } \\
\text { aparatos del hogar }\end{array}$ & 0.54 & 0.015 & 2.783 & 0.07 & 0.010 & 0.994 & 0.05 & 0.006 & 0.984 & \\
\hline $\begin{array}{l}\text { Horas trabajo } \\
\text { remunerado }\end{array}$ & 48.36 & 0.084 & 15.786 & 38.34 & 0.168 & 16.918 & 35.83 & 0.110 & 17.271 & * \\
\hline Ingreso por hora & 27.31 & 0.217 & 41.514 & 26.47 & 0.441 & 45.380 & 26.95 & 0.273 & 43.516 & \\
\hline $\begin{array}{l}\text { Sin seguridad social } \\
\text { por empleo }\end{array}$ & 54.93 & 0.003 & 0.498 & 61.91 & 0.005 & 0.486 & 62.55 & 0.003 & 0.484 & \\
\hline Edad & 40.17 & 0.056 & 10.781 & 48.42 & 0.116 & 11.949 & 42.22 & 0.068 & 10.819 & $*$ \\
\hline Años de escolaridad & 9.72 & 0.027 & 5.165 & 9.07 & 0.055 & 5.698 & 10.09 & 0.033 & 5.310 & $*$ \\
\hline $\begin{array}{l}\text { Número de } \\
\text { personas en el hogar }\end{array}$ & 4.78 & 0.008 & 1.601 & 3.24 & 0.017 & 1.780 & 4.30 & 0.010 & 1.542 & $*$ \\
\hline Número de hijos & n.d. & n.d. & n.d. & 3.13 & 0.02 & 2.01 & 2.87 & 0.01 & 1.64 & $*$ \\
\hline
\end{tabular}

* Prueba $t$ de dos muestras con varianzas iguales significativas.

n.d.: información no disponible.

Fuente: Elaboración propia con base en INEGI, ENOE, trimestre I de 2015. 
Las tendencias presentadas en esta sección confirman que existen diferencias en el número de horas dedicadas al trabajo doméstico entre madres esposas y madres jefas de hogar. El segundo paso de nuestro análisis tiene como objetivo comprender la relación entre esta carga de trabajo doméstico y las características demográficas de estas poblaciones.

\section{Determinantes del trabajo doméstico}

En esta parte del análisis se excluyó a los varones para comparar solo los patrones de trabajo doméstico de las poblaciones de mujeres. El cuadro 2 muestra los valores descriptivos de las muestras.

CUADRO 2. Estadísticos descriptivos de las muestras. Porcentajes ajustados por el factor de expansión

\begin{tabular}{|c|c|c|}
\hline $\begin{array}{c}\text { Características } \\
\text { seleccionadas }\end{array}$ & $\begin{array}{c}\text { Madres jefas de } \\
\text { hogar }\end{array}$ & $\begin{array}{l}\text { Madres } \\
\text { esposas }\end{array}$ \\
\hline \multicolumn{3}{|l|}{ Horas de trabajo remunerado semanales } \\
\hline Menos de 15 (ref.) & 11.2 & 15.7 \\
\hline Entre 15 y 34 & 23.3 & 24.7 \\
\hline Más de 35 & 65.6 & 59.7 \\
\hline \multicolumn{3}{|l|}{ Condición de ocupación } \\
\hline Comerciantes & 24.7 & 28.6 \\
\hline Profesionales, técnicos y trabajadores del arte & 6.3 & 7.7 \\
\hline Trabajadores de la educación & 4.3 & 6.2 \\
\hline Funcionarios y directivos & 1.8 & 1.9 \\
\hline Oficinistas & 10.2 & 9.4 \\
\hline Trabajadores industriales artesanos y ayudantes & 17.6 & 19.1 \\
\hline Operadores de transporte & 0.1 & 0.1 \\
\hline Trabajadores en servicios personales & 30.9 & 23.0 \\
\hline Trabajadores en protección y vigilancia & 0.4 & 0.2 \\
\hline Trabajadores agropecuarios & 3.9 & 3.8 \\
\hline \multicolumn{3}{|l|}{ Acceso a seguridad social por empleo } \\
\hline Sí & 33.7 & 32.5 \\
\hline No & 66.3 & 67.5 \\
\hline
\end{tabular}


Educación

Sin educación

$$
8.6
$$

Primaria

Secundaria

Media superior

Grupo de edad

De 15 a 19 años

De 20 a 29 años

De 30 a 39 años

De 40 a 49 años

De 50 a 59 años

De 60 años y más

Con trabajador doméstico en el hogar

Hijos

Media

$3.2 \quad 3.0$

Desviación estándar

Mínima

Máxima

Personas en el hogar

Media

Desviación estándar

$1.8 \quad 1.6$

Mínima

1.0

2.0

Máxima

22.0

Tamaño de la muestra

10,538 26.0

Tamaño de la población

Fuente: Elaboración propia con base en INEGI, ENOE, trimestre I de 2015.

En primera instancia, se observa que en la categoría de más de 35 horas de trabajo remunerado semanal la proporción de madres jefas es mayor que la de madres esposas. Por otra parte, la principal ocupación donde trabajan las madres jefas es la de servicios personales (que comprende salones y clínicas de belleza, baños y sanitarios públicos, lavanderías, tintorerías, estacionamientos, servicios funerarios, servicios de revelado fotográfico, entre otros) con $30.9 \%$; seguida por la ocupación de comerciante, que puede ser en el sector formal o el informal, y en tercer lugar, estas madres jefas trabajan en la industria como obreras o artesanas (17.6\%). En el caso de las madres esposas la principal ocupación donde se encuentran es la de 
comerciantes con $28.6 \%$, seguida con $23 \%$ por servicios personales y en tercer lugar la misma que en las madres jefas, como trabajadoras industriales, artesanas y ayudantes $(19.1 \%)$. En el caso de las mujeres en posiciones altas como funcionarias y directivas se encontró que solo el $1.8 \%$ y $1.9 \%$, respectivamente, de ellas se encuentran en estas ocupaciones, estos resultados están de acuerdo con lo hallado por Pedrero Nieto (2009). En la tabla se muestra que la precariedad laboral en que se encuentran trabajando se identifica con la ausencia de seguridad social, la cual asciende a $66.3 \%$ y $67.5 \%$ respectivamente. La proporción de mujeres con un empleado doméstico, que puede ser de planta o de entrada por salida, es de $3.9 \%$ para las madres jefas de hogar y de $6.5 \%$ para las madres esposas. Aunque el valor de esta proporción es mayor para las madres esposas, se puede considerar bajo y que no influye de manera importante en la carga de trabajo doméstico, pues ellas reportaron una mayor carga de horas.

Después de observar las estadísticas descriptivas, es necesario hacer un análisis más sofisticado a través de una regresión lineal. A este respecto, como se explicó en la sección de métodos, las horas dedicadas al trabajo doméstico fueron estandarizadas con el fin de que fueran comparables e interpretables entre ambas poblaciones. Una limitación notable de la fuente de información es que permite identificar si las mujeres tienen o no hijos; sin embargo, no proporciona la edad de estos. Al considerar clave la información acerca de la edad de los hijos, se aproximó este valor con información de los hogares acerca de la presencia de niños de cero a seis y de siete a doce años, los cuales es probable que sean los hijos de las madres de la muestra.

CUADRO 3. Coeficiente de regresión de las horas de trabajo doméstico estandarizadas

Características seleccionadas

Horas de trabajo semanales

Menos de 15 (ref.)

Entre 15 y 34

Más de 35

Condición de ocupación

Comerciantes (ref.)

Profesionales, técnicos y trabajadores del arte

Trabajadores de la educación

Funcionarios y directivos

Oficinistas

Trabajadores industriales artesanos y ayudantes

\begin{tabular}{rrrr} 
Madres jefas de hogar & \multicolumn{2}{c}{ Madres esposas } \\
Coeficiente & $p>|t|$ & Coeficiente & $p>|t|$ \\
& & & \\
-0.156 & $\star$ & -0.221 & $\star$ \\
-0.342 & $\star$ & -0.535 & $\star$
\end{tabular}

$-0.008$

$-0.081$

$-0.025$

$-0.040$

$-0.197$

$-0.222$

0.014

$-0.071$

0.007 
¿Es la doble jornada igual para todas? Distribución y factores determinantes del trabajo doméstico... \|| ANDREA BAUTISTA LEÓN \& GABRIELA SánCHEZ SoTO

Operadores de transporte

Trabajadores en servicios personales

Trabajadores en protección y vigilancia

Trabajadores agropecuarios

Acceso a seguridad social por empleo

Sí (ref.)

No

\section{Educación}

Secundaria (ref.)

Sin educación

Primaria

Media superior

Superior y posgrado

Grupo de edad

40 a 49 años (ref.)

De 15 a 19 años

De 20 a 29 años

De 30 a 39 años

De 50 a 59 años

De 60 años y más

Con trabajador doméstico en el hogar

Hogar con niño menor de 6 años

Hogar con menor entre 7 y 12 años

Persona mayor de 18 años no hijo

Tamaño de la muestra

Tamaño de la población

Prob $>F$

$R$-cuadrada

${ }^{*} p<0.05 ;{ }^{* *} \mathrm{p}<0.10$
$-0.059$

$-0.446$

$-0.014$

$-0.073$

0.213

$-0.289$

0.050

$-0.044$

0.058

0.092

$\begin{array}{lll}0.022 & -0.008 \\ 0.030 & & 0.001 \\ 0.055 & 0.124 \\ 0.086 & * * & \\ & & \end{array}$

0.302 * 0.414

0.168 * 0.233

$\begin{array}{lll}-0.070 & * & -0.163\end{array}$

$\begin{array}{lll}-0.220 & * & -0.265\end{array}$

-0.257 * $\quad-0.264$

0.115 * 0.171

0.155 * 0.135

$-0.105$

$-0.140$

25,227

10,538

$7,152,309$

0.00

0.00

0.12

0.22

Fuente: Elaboración propia con base en INEGI, ENOE, trimestre I de 2015. Resultados ajustados por el diseño de la encuesta. 
El cuadro 3 contiene los resultados de las regresiones estimadas. Uno de los determinantes principales en la cantidad de trabajo doméstico es el número de horas de trabajo extradoméstico. En este caso se codificó una variable que identifica si el trabajo extradoméstico en la semana de referencia tuvo una duración menor de 15 horas, de 15 a 34 horas o de más de 35 horas. El grupo de referencia fue el trabajo remunerado de menos de 15 horas a la semana, y los resultados sugieren que jornadas de trabajo extradoméstico mayores de 15 horas reducen la cantidad de tiempo dedicado al trabajo doméstico para ambas poblaciones. Sin embargo, el efecto de reducción en ambas categorías es más pronunciado para las madres solteras $(-0.156$ y -0.342) que para las madres esposas (-0.221 y -0.535).

Para las ocupaciones se utilizó el grupo de comerciantes como referente por la proporción importante que tiene en ambas poblaciones. A este respecto resulta interesante que para las madres jefas solo la ocupación de funcionarias y directivas fue significativa y demostró que causa una reducción en la carga de trabajo doméstico, mientras que para las madres esposas una variedad más amplia de ocupaciones resulto significativa, pues causan todas ellas una disminución en la carga de trabajo. En orden de magnitud, la diferencia negativa más grande con respecto a las comerciantes fue la de trabajadoras en protección y vigilancia $(-0.289)$, seguida por funcionarias y directivas (-0.222), profesionistas $(-0.081)$, servicios personales $(-0.073)$, oficinistas $(-0.071)$ y finalmente por las trabajadoras en la industria $(-0.068)$. En el caso de las dos primeras ocupaciones mencionadas se explica que la diferencia sea mayor por la naturaleza de estos trabajos, que requieren pasar muchas horas fuera de casa, con lo cual el tiempo efectivo para estar en el hogar y realizar trabajo doméstico se vuelve mínimo.

El que las mujeres que se dedican a los servicios personales tengan una diferencia más pequeña respecto del grupo de referencia (comerciantes) no es de sorprender, pues muchas mujeres que se dedican a este tipo de actividades en el sector servicios suelen llevar a los hijos al trabajo. Entonces, los límites entre la carga de trabajo doméstico y la del trabajo remunerado se vuelven confusos.

Como se mencionó en el análisis anterior, el nivel educativo está asociado positivamente con la cantidad de trabajo doméstico que las mujeres realizan (Casique, 2008). En este caso, después de controlar por otras variables, para ambas poblaciones se encontró este efecto, tener más escolaridad está asociado con una carga en el trabajo doméstico.

Con respecto a la edad de las mujeres, se conoce por la estimación de la diferencia de medias mostrada en el cuadro 1 que las madres jefas son en promedio seis años mayores que las madres cónyuges. En ambas poblaciones el promedio de edad se encuentra en el grupo de 40 a 49 años. Con esta última información, se decidió usar a este grupo como referencia en el modelo de regresión lineal. Lo que se observa en el cuadro 3 es que para ambas poblaciones en los grupos más jóvenes que para el grupo de referencia (entre 15 y 39 años) la carga de trabajo doméstico 
es mayor. La magnitud de los coeficientes es mayor para las madres esposas, lo cual está relacionado con la estructura de edad de este grupo de madres, esto es, estas son más jóvenes que las madres jefas. Los coeficientes para grupos de edad mayores de 50 años indican que estas mujeres realizan menos trabajo doméstico si se les compara con el grupo de referencia para ambas poblaciones.

La presencia de niños menores de seis años y de entre siete y doce años en el hogar tiene un efecto de incremento en la carga de trabajo doméstico. La magnitud de este efecto es diferente entre estas poblaciones, siendo para las madres jefas mayor la carga de niños de entre siete y doce años que para las madres esposas de niños menores de seis años. Este resultado era esperado y es atribuible a la diferencia en el promedio de edad entre estas poblaciones, y por ende a la edad de sus hijos.

Ambos resultados previos indican que la edad de las madres tiene sin duda una influencia en la magnitud y la distribución de carga de trabajo doméstico que realizan, además del efecto relacionado con la edad de sus hijos.

Finalmente, se conoce que algunas de las madres jefas habitan en hogares extensos o bien se apoyan con otros adultos principalmente para el cuidado de sus hijos (Álvarez \& Gómez, 2011; Castellanos Lázaro, Zapata Martelo \& Martínez Corona, 2007), por lo cual se incluyó una variable que identificó a los individuos mayores de 18 años que no fueran hijos de la jefa o bien de la cónyuge, en el caso de las madres esposas, para conocer el efecto que tiene sobre el trabajo doméstico. Los resultados demuestran que la presencia de un adulto en el hogar disminuye la carga de trabajo doméstico, el efecto de disminución fue ligeramente mayor para las madres jefas que para las madres esposas.

\section{Discusión y conclusión}

La realización del trabajo doméstico es necesaria para el mantenimiento de todos los miembros de los hogares. Sin embargo, es una tarea que en la actualidad sigue siendo reconocida mayormente como femenina, lo cual causa un conflicto en el balance de los diferentes roles en que las mujeres actuales se encuentran, como madres, esposas y empleadas. Esta investigación planteó tres preguntas principales acerca de la dinámica del trabajo global entre madres esposas y madres jefas de hogar. La primera buscaba conocer la diferencia en la carga de trabajo global entre ellas. Los resultados indican que, si se consideran las horas totales dedicadas a ambas labores, las madres jefas dedican 65.0 horas a la semana, mientras que las madres esposas emplean 69.3 horas, resultados que concuerdan con la tendencia observada por Pedrero Nieto (2004) y establece una diferencia de cuatro horas extra semanales de trabajo global que realizan las madres esposas. Una vez establecida esta diferencia, para responder la segunda pregunta, acerca de los determinantes 
sociodemográficos de la carga de trabajo, destaca en los resultados que un mayor grado de educación tiene una relación positiva con la carga de trabajo doméstico, y que la edad de las mujeres está relacionada con una mayor o menor carga. Esto significa, cuando se les compara a las mujeres con el grupo de edad media para ambas poblaciones de esta muestra ( 40 a 49 años), que las más jóvenes tienen más horas de trabajo doméstico que las de más de 50 años. Lo anterior está ligado también con la edad de los hijos de ambas poblaciones; puesto que las madres esposas son en promedio seis años más jóvenes que las jefas, es probable que sus hijos sean pequeños y requieran más tiempo de cuidados. Estos resultados indican que la edad y la etapa en el ciclo de vida en el que se encuentran las madres y sus hijos es un determinante primordial de la carga de trabajo, lo cual llama a reflexionar acerca de la participación de los padres en el tiempo de cuidado de los hijos para aliviar la carga de trabajo de las madres esposas. Como se mostró en el cuadro 1, los hombres jefes que habitan en hogares con niños dedican en promedio 12.2 horas semanales menos que sus pares mujeres. Por otra parte, contar con un trabajador doméstico y la presencia de otro adulto en el hogar disminuye esta carga. La tercera pregunta que se planteó es si existe diferencia en la carga de trabajo doméstico por el tipo de ocupación. A este respecto, primero, los resultados mostraron mayor significancia estadística para las madres esposas, que todas las ocupaciones reducen la carga de trabajo doméstico y que hay magnitudes distintas de acuerdo con el tipo de ocupación y están relacionadas con la naturaleza del trabajo y su localización. Esto es, las actividades que les requieren a las madres estar fuera del hogar y a las cuales no es posible llevar a los hijos disminuyen de manera más sustantiva su carga doméstica. Aunado a lo anterior, las condiciones laborales importan; esto es, las condiciones precarias, sin seguridad social, influyen en un detrimento del bienestar de las madres trabajadoras causándoles mayor carga de trabajo doméstico.

A manera de conclusión, este ejercicio de comparación entre madres esposas y madres jefas y su carga de trabajo global hace un llamado a seguir analizando a la población de mujeres por características especiales, por una parte. Por otra, remarcamos la importancia de seguir incluyendo en la agenda de investigación el estudio de los mecanismos de acceso al poder y la toma de decisiones de las mujeres en los hogares, los efectos de las desigualdades en la carga de trabajo en la salud física y mental, así como en la eliminación de las presiones sociales en torno al ideal de familia y a la participación femenina en el trabajo remunerado.

\section{Referencias}

Álvarez, A. \& Gómez, I. (2011). Conflicto trabajo-familia, en mujeres profesionales que trabajan en la modalidad de empleo. Pensamiento Psicológico, 9(16), 89-106. 
Baxter, J. \& Tai, T. (2016). Inequalities in unpaid work: a cross-national comparison. En Connerley, M. \& Wu, J. (eds.), Handbook on well-being of working women (pp. 653671). Springer.

Buchmann, M., Kriesi, I., Pfeifer, A. \& Sacchi, S. (2003). Labor-market opportunities and women's labor-market exit and reentry. En Heinz, W. \& Marshall, V. (eds.), Social dynamics of the life course: transitions, institutions, and interrelations (pp. 117-141). New York: Aldine de Gruyter.

Casique, I. (2008). Participación en el trabajo doméstico de hombres y mujeres en México. Papeles de Población, 14(55), 173-200.

Castellanos Lázaro, R., Zapata Martelo, E. \& Martínez Corona, B. (2007). Jefas de hogar: cambios en el trabajo y en las relaciones de poder. Política y Cultura, 28, 194-210.

Connerley, M. L. \& Wu, J. (2016). Uncovering the complexities of the relationship between women and well-being in the workplace: an introduction. En Connerley, M. L. \& Wu, J. (eds.), Handbook on well-being of working women (pp. 3-13). Springer.

Cuevas Hernández, A. (2010). Jefas de familia sin pareja: estigma social y autopercepción. Estudios Sociológicos, 28(84), 753-789.

Durán, M. A. (2000). Concentración y reparto del trabajo no remunerado en los hogares. Cuaderno de Relaciones Laborales, 17, 91-122.

Farías Anda, S., Salazar Segura, M., Monjardin Nieto, F. \& Calleja Bello, N. (2014). Jefas de familia en México: caracterización y rasgos instrumentales-expresivos. Revista Mexicana de Investigación en Psicología, 6(1), 87-96.

García, B. \& Pacheco, E. (2000). Esposas, hijos e hijas en el mercado de trabajo de la Ciudad de México. Estudios Demográficos y Urbanos, 43, 35-63.

Guarriello Heath, R. (2016). "Women like you keep women like me down": understanding intergenerational conflict and work-life balance from a discourse perspective. En Connerley, M. L. \& Wu, J. (eds.), Handbook on well-being of working women (pp. 65-82). Springer.

Hochschild, A. \& Machung, A. (2012). The second shift. Working families and the revolution at home (3rd ed.). New York: Penguin.

Instituto Nacional de Estadística y Geografía (INEGI) (2007). Cómo se hace la ENOE. Métodos y procedimientos. Recuperado de http://www.beta.inegi.org.mx/app/biblioteca/ ficha.html?upc $=702825006541$

Instituto Nacional de Estadística y Geografía (INEGI) (2010). Serie histórica censal e intercensal. Recuperado de http://www.inegi.org.mx/est/contenidos/Proyectos/ccpv/ cpvsh/Series_historicas.aspx

Instituto Nacional de Estadística y Geografía (INEGI) (2016). Cuéntame matrimonios y divorcios. Recuperado de http://cuentame.inegi.org.mx/poblacion/myd.aspx?tema=P

Jociles, M., Monco, B., Villamil, F. \& Díaz, P. (2008). Una reflexión crítica sobre la monoparentalidad: el caso de las madres solteras por elección. Portularia, viII(1), 265-278. Nicholson, J. (2014). t-test. (O.U. Press, ed.) Recuperado de http://www.oxfordreferen- 
ce.com.libweb.lib.utsa.edu/view/10.1093/acref/9780199679591.001.0001/acref9780199679591-e-2897

Ochoa Ávalos, M. C. (2007). Pobreza y jefatura femenina. La Ventana, 25, 168-198.

Oliveira, O. \& García, B. (2004). Mujeres jefas de hogar y su dinámica familiar. I Congresso da Associaçao Latino Americana de Populaçao (pp. 1-18). Caxambu, MG Brasil: ALAP.

Pedrero Nieto, M. (2004). Género, trabajo doméstico y extradoméstico en México. Una estimación del valor económico del trabajo doméstico. Estudios Demográficos y Urbanos, 19(2), 413-446.

Pedrero Nieto, M. (2009). Las condiciones de trabajo a principios del siglo xxi. Presencia de las mujeres en el sector informal. Papeles de Población, 15(59), 119-171.

Salinas Jiménez, M., Artés, J. \& Salinas Jiménez, J. (2016). Are educational and occupational status affecting men's and womens satisfaction in different ways? A cross-national perspective on gender inequality. En Handbook on well-being of working women (pp. 603-632). Springer.

Santoyo, L. \& Pacheco, E. (2014). El uso del tiempo de las personas en México según tipo de hogar. Una expresión de las desigualdades de género. En García, B. \& Pacheco, E. (coords.), Uso del tiempo y trabajo no remunerado en México (pp. 171-220). Ciudad de México: El Colegio de México.

Tobio Soler, C. (2002). Conciliación o contradicción: cómo hacen las madres trabajadoras. Revista Española de Investigaciones Sociológicas, 97, 155-186.

Valdivia Sánchez, C. (2008). La familia: concepto, cambios y nuevos modelos. La Revue du REDIF, 1, 15-22.

Zabludovsky, G. (2007). Las mujeres en México: trabajo, educación superior y esferas de poder. Política y Cultura, 28, 9-41. 